\title{
D’Hypo-Proust en Hyper-Proust ?
}

Les « brouillons » imprimés de l'édition électronique

Nathalie Mauriac Dyer

\section{OpenEdition}

\section{Journals}

Édition électronique

URL : http://journals.openedition.org/recherchestravaux/103

DOI : 10.4000/recherchestravaux.103

ISSN : 1969-6434

Éditeur

UGA Éditions/Université Grenoble Alpes

Édition imprimée

Date de publication : 15 juin 2008

Pagination : 157-170

ISBN : 978-2-84310-125-0

ISSN : 0151-1874

Référence électronique

Nathalie Mauriac Dyer, « D'Hypo-Proust en Hyper-Proust ? », Recherches \& Travaux [En ligne], 72 | 2008, mis en ligne le 15 décembre 2009, consulté le 03 février 2021. URL : http://journals.openedition.org/ recherchestravaux/103; DOI : https://doi.org/10.4000/recherchestravaux.103 


\section{D’Hypo-Proust en Hyper-Proust? Les «brouillons » imprimés de l'édition électronique}

Publier des cahiers manuscrits n'est nullement publier «l'œuvre' $»$ d'un auteur, mais quelques-unes des traces de sa fabrique : c'est donc une entreprise qui se distingue, au moins en partie, de celle, bien balisée par des siècles de tradition, de l'édition de texte, et qui exige la définition et la mise au point de protocoles éditoriaux spécifiques. À l'heure de la révolution numérique, ces protocoles doivent-ils être électroniques? Activement engagée dans un projet d'édition imprimée au long cours, mais aussi à plus court terme dans une entreprise de publication en ligne, $j$ 'essaierai, après une brève contextualisation historique de l'édition des manuscrits proustiens, de montrer qu'il n'y a pas, dans le domaine qui est le mien en tout cas, dilemme ni conflit, mais plutôt complémentarité, voire interdépendance, des deux approches. Je risquerai même l'idée que l'édition imprimée en cours dont je vais présenter les grandes lignes, celle des cahiers, constitue ce qu'on pourrait appeler l'«HypoProust», c'est-à-dire l'indispensable soubassement de connaissances sans

I. Même si longtemps publier des cahiers manuscrits de Proust signifia, pour ses éditeurs, publier une partie de son «œuvre» : ainsi en 1925 Albertine disparue et en 1927 Le Temps retrouvé, considérés comme les derniers «tomes » d'À la recherche du tempsperdu mais tirés de cahiers de la série dite de «mise au net» rédigés à partir de I9I 5 (Cahiers XII-XX); ou en I 952 et I 97 I, les deux versions de Contre Sainte-Beuve, montages d'extraits des cahiers de I 908-I 909. Aujourd'hui encore, pour le «grand» public et le public cultivé (sinon pour bien des spécialistes), ces différents volumes posthumes sont tenus pour d'authentiques œuvres de Proust, au même titre que les «anthumes», qui seuls relèvent de plein droit du régime d'auctorialité (Les Plaisirs et les jours, Du côté de chez. Swann ou Pastiches et mélanges, par exemple). 
lequel il serait impossible d'envisager un jour la mise en œuvre de l'«HyperProust» électronique. C'est à partir de l'Hypo-Proust qu'on peut commencer à s'interroger sur la singularité de l'hypertexte génétique proustien, et sur les modalités de sa mise en œuvre électronique.

\section{L'édition des manuscrits proustiens au $\mathrm{XX}^{\mathrm{e}}$ siècle}

Dès I 976, soit une quinzaine d'années après la constitution du «fonds Proust», la publication des manuscrits proustiens avait été envisagée par les spécialistes, fédérant une équipe du CNRS autour d'un projet éditorial chez Gallimard : "édition en une douzaine de volumes de L'Atelier de Proust, c'est-à-dire de l'ensemble des esquisses et des ébauches de $\dot{A}$ la recherche $d u$ temps perdu principalement contenues dans les soixante-deux cahiers de brouillon qui figurent depuis 1962 au fonds Proust de la Bibliothèque nationale ${ }^{2} »$. Cette édition - qui se voulait la première étape d'une «édition d'ensemble des "avant-textes" de la Recherche» (ibid.) - ne vit jamais le jour. Mais le système de transcription élaboré pour L'Atelier de Proust se généralisa auprès des spécialistes. Tous azimuts et en ordre dispersé, un «corpus» de transcriptions linéarisées se constitua peu à peu, dans des périodiques spécialisés, de nombreuses thèses, voire en volume - qu'on songe à l'importante édition des Cahiers 5 I, 57 et 58 procurée par Henri Bonnet et Bernard Brun 3 , puis aux «Esquisses» réunies à la fin de chacun des quatre volumes d'A la recherche du temps perdu de la récente édition du roman proustien dans la «Bibliothèque de la Pléiade»4. L'inventaire réalisé en I99 I' a montré, toutefois, que s'était ainsi constitué moins un «corpus» qu'un ensemble de membra disjecta, chacun ou presque des soixante-quinze cahiers de brouillon ${ }^{6}$ ayant fait l'objet de transcriptions de la part de trois ou quatre chercheurs, sans que jamais, ou presque, un cahier ait été intégralement transcrit ${ }^{7}$, alors que les fragments transcrits plusieurs fois ne sont pas rares.

2. Voir Bulletin d'informations proustiennes, $\mathrm{n}^{\circ}$ 4, automne i976, p. 7, J. Bersani pour l'équipe Proust.

3. M. Proust, Matinée chez la princesse de Guermantes : Cabiers du Temps retrouvé, édition critique établie par H. Bonnet en collaboration avec B. Brun, Gallimard, I 982.

4. M. Proust, $\grave{A}$ la recherche du temps perdu, édition publiée sous la direction de J.-Y. Tadié, Gallimard, «Bibliothèque de la Pléiade», 4 volumes, I987-1989.

5. N. Sebban, «Liste des inédits proustiens publiés», Bulletin d'Informations proustiennes, $\mathrm{n}^{\circ} 22$; inventaire complété dans les livraisons suivantes.

6. Treize nouveaux cahiers sont entrés au fonds Proust en I984.

7. Les rares transcriptions intégrales existant, toujours dans des thèses (Th. Laget [1984], F. Goujon [1996]), sont demeurées inédites. 
Qui, d'autre part, comparerait les protocoles employés constaterait, à côté de transcriptions linéarisées s'efforçant à l'exhaustivité, d'autres (comme celles qu'on trouve dans la «Bibliothèque de la Pléiade») revendiquant leur caractère allégé de toutes les traces du travail rédactionnel, en particulier de tous les passages biffés ${ }^{8}$. La conséquence, pour les études proustiennes de genèse, n'est pas anodine : leur corpus, tout simplement, n'est pas disponible. Le chercheur doit pour chaque cahier qui l'intéresse réunir, vérifier et compléter les fragments transcrits, avant de pouvoir valider les hypothèses existantes et en formuler de nouvelles. Pour le proustien non "généticien», grande est la tentation de se contenter du riche contenu thématique offert par les «Esquisses», même si elles ne sont qu'un succédané des manuscrits de référence.

\section{L'édition des manuscrits proustiens au XxI siècle : le projet d'édition des Cahiers I à 75 de la Bibliothèque nationale de France, BnF-Brepols}

L'objectif de l'ambitieux projet éditorial des Cahiers - plus de sept mille pages manuscrites confiées à une trentaine de spécialistes 9 - est de produire le corpus central des études proustiennes de genèse, corpus qui deviendra du même coup disponible pour d'autres domaines d'étude. Le protocole de cette édition imprimée, tout en se réclamant du meilleur de la tradition philologique (rigueur dans l'établissement du «texte», annotation historicocritique exhaustive), présente des spécificités.

- La première, qui a fait ses preuves dans d'autres corpus, est le couplage de la transcription diplomatique intégrale de chaque cahier avec son facsimilé; pour faciliter la consultation, transcription et fac-similé (en couleur, à l'échelle, et établi d'après la numérisation de la Bibliothèque nationale de France) seront présentés dans deux volumes séparés.

- On proposera ensuite le diagramme de chaque cahier, c'est-à-dire la représentation schématique, sur des vignettes du fac-similé, des «unités textuelles» le constituant. Ces diagrammes ou «cartes» (fig. I) ont pour but de guider le lecteur dans la topographie parfois labyrinthique de l'écriture proustienne; ils ne prétendent pas élucider l'ordre dans lequel les unités textuelles

8. Voir l'affirmation paradoxale des éditeurs : le «but n'est $[\ldots]$ pas de permettre de lire les cahiers de brouillons de Proust» (À la recherche du tempsperdu, op. cit., t. I, I987, p. CLXXIV, note I). 9. Le comité éditorial responsable comprend B. Brun, A. Compagnon, P.-L. Rey, K. Yoshikawa, et N. Mauriac Dyer (directeur). Le Cahier 54, F. Goujon, N. Mauriac Dyer et C. Nakano éd., est à paraître en 2008. 

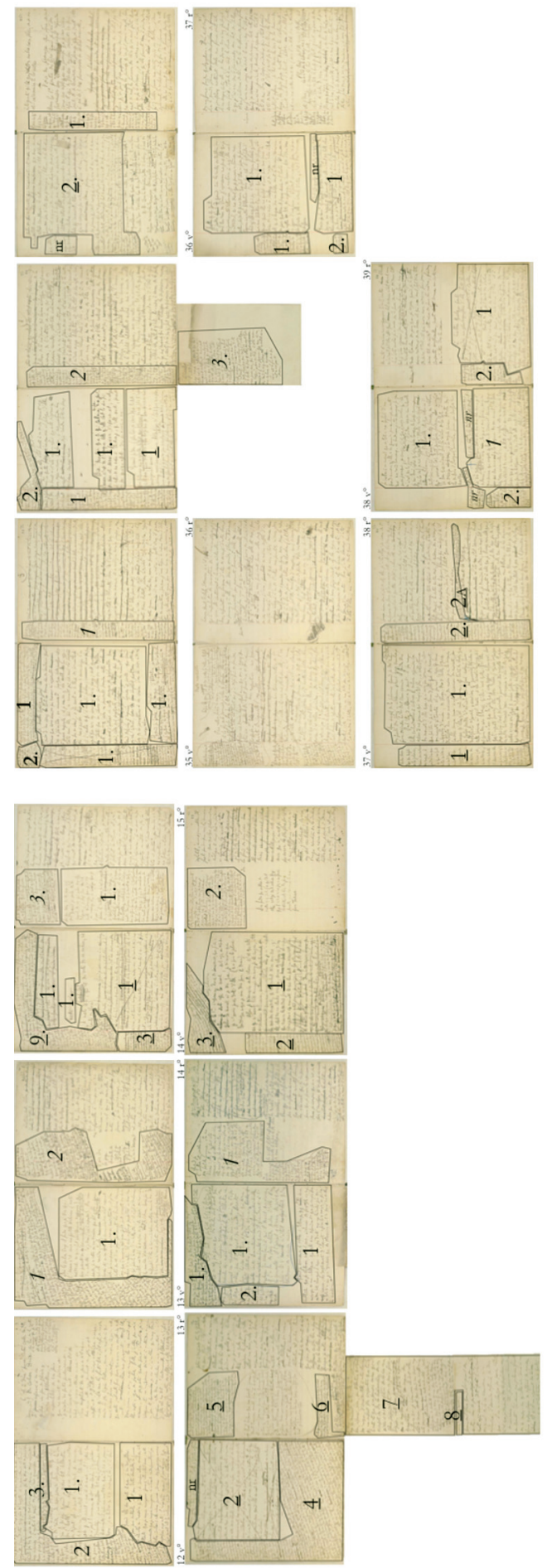

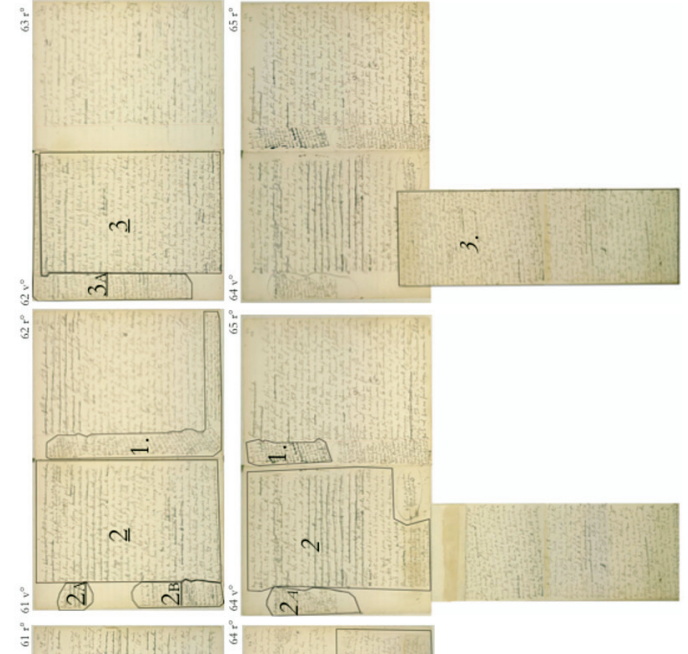

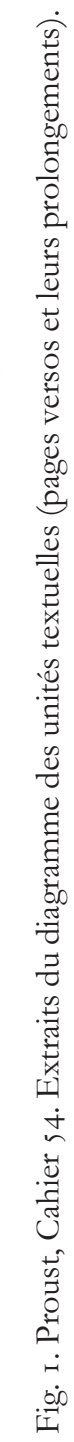


en question ont été rédigées, mais distinguent, pour plus de clarté, les unités textuelles rédigées sur les rectos (ou «lignes principales») du cahier, de celles rédigées sur les versos ou dans les marges des rectos, qui sont en principe plus tardives ${ }^{10}$.

- On proposera, en sus de l'annotation historico-critique, une annotation génétique, dont l'objet sera de replacer autant que possible le cahier, folio par folio, dans son contexte dynamique, celui de la rédaction du roman. Il s'agira en particulier de signaler les passages - des unités étendues sur plusieurs pages, quelques phrases ou quelques mots - que Proust a empruntés à de précédents manuscrits, et ceux qu'il a ensuite introduits ailleurs, tout en commentant le type de modification intervenu : copieadaptation, développement-amplification, montage-regroupement, éclatement-dissémination, changement de contexte, transfert sur un autre personnage, fait de style, etc. L'annotation génétique consistera aussi à repérer, inversement et de façon complémentaire, non les trajets mais les impasses, les culs-de-sac, les abandons, les hapax - et les lacunes. L'enjeu - énorme! - est de réaliser un relevé aussi complet que possible, cahier par cahier, des milliers d'événements d'écriture dont le corpus des Cahiers I à 75 porte la trace.

- Enfin, à partir de cette annotation, on établira cahier par cahier un «index des renvois génétiques», élément décisif du dispositif éditorial dans la perspective, précisément, d'une solidarité entre supports d'édition. Cet index comportera plusieurs rubriques. Il recensera tout d'abord les cahiers et manuscrits cités par Proust, de manière explicite ou allusive, dans le cahier édité; puis les autres manuscrits de Proust citant ce cahier. Enfin, une troisième rubrique, de loin la plus fournie, recensera tous les cahiers - et précisément dans ces cahiers tous les folios - cités dans les notes à propos d'un (ou plusieurs) folio(s) du cahier de référence. Cette rubrique de l'index permettra donc de saisir en un clin d'œil le «périmètre» génétique, si l'on veut, d'un cahier donné; pour le Cahier 54, par exemple, on constate que pas moins de trente-neuf autres cahiers du corpus des Cahiers I à 75 ont dû être convoqués pour établir l'annotation génétique. L'index des renvois génétiques constitue ainsi, dans toutes ses dimensions, la base d'une cartographie fine des réécritures, quasiment chaque folio du cahier indexé étant «relié» génétiquement, au moins en partie, à tel(s) ou tel(s) folio(s) d'un (voire plusieurs) autre(s) cahier(s).

I o. Plus complexes sont les cas (pas si rares) où Proust utilise son cahier à la fois sur l'endroit et sur l'envers : les versos sont alors utilisés comme des rectos. 


\section{Horizons électroniques à court terme : répertoires, hyper-index, dia- grammes génétiques}

L'édition imprimée va ainsi constituer peu à peu un réservoir pérenne et aisément accessible de connaissances, dont le contenu pourra (devra) toutefois être régulièrement mis à jour, puisque l'annotation génétique, en particulier, aura probablement été lacunaire, en particulier dans les premiers cahiers publiés. Diverses publications annexes - qui gagneront à être élaborées en ligne - peuvent d'ores et déjà être envisagées :

- au fur et à mesure de la publication, on pourra compilerles index des renvois génétiques pour constituer progressivement d'une part un Répertoire des cabiers cités et citant, d'autre part l'Hyper-index qui balisera non seulement les trajets vers les, et à partir des, folios d'un cahier donné, mais entre les folios des divers (de tous les cahiers, sans privilégier une «tête de pont» du réseau ${ }^{11}$.

- Comme des diagrammes cartographient les unités textuelles pour guider le lecteur, d'autres diagrammes pourront être constitués, qui, sur des vignettes du fac-similé et/ou de la transcription diplomatique, définiront, pour chaque folio, un zonage par unités génétiques pertinentes. La provenance, ou la destination, de ces unités serait indiquée dans la marge. Pour l'étude de genèse, la granularité pertinente est en effet à géométrie variable : s'il y a des «cahiers Sainte-Beuve», des «cahiers des Jeunes filles» ou des «cahiers Guermantes», le généticien raisonne de toute évidence à une échelle plus fine, par mise en relation ou en contraste non seulement d'unités textuelles, mais aussi de folios et, très fréquemment, de zones de folios : les diagrammes génétiques les mettront précisément en évidence.

En point de mire se trouve donc la cartographie, assez vertigineuse, de ce qu'il faut bien appeler l'hypertexte génétique du roman proustien dans les Cahiers I à 75 - étant bien entendu que l'information fournie par les cahiers ne donne accès qu'à une phase et non à l'intégralité du processus génétique, qui chez Proust se poursuit, et avec intensité, sur les dactylographies et les placards d'imprimerie.

Dans l'édition imprimée toutefois, et même dans l'édition imprimée avec ses premiers compléments électroniques, cet hypertexte génétique restera encore statique, «virtuel» si l'on préfère, tant que le lecteur n'aura pas «activé» les liens repérés dans l'annotation, recensés par les index et l'hyper-index et

I I. Il va de soi qu'on peut (doit) aussi envisager un hyper-index nominum (noms de personnes, personnages, lieux, œuvres). 
figurés par les diagrammes génétiques en se reportant effectivement aux (zones des) folios des cahiers cités pour les comparer aux (zones des) folios du cahier de référence - et en tirer les diverses conclusions, esthétiques, stylistiques, etc., qui s'imposent à lui. Ici, une traduction électronique immédiate serait évidemment précieuse en accélérant cet accès aux folios et zones de folios considérés. Mais encore faudrait-il qu'ait été résolue la question préalable : sous quelles formes concevoir l'hypertexte, et donc quelle interface donner à l'Hyper Proust? Prenons un exemple.

\section{Problèmes de représentation d'une «figure de genèse" proustienne : la dissémination}

S'il y a des «styles» singuliers de genèse ${ }^{12}$, on peut peut-être parler aussi, comme il y a des figures de rhétorique, de «figures» génétiques. L'une d'elles, caractéristique du style proustien de genèse et familière aux spécialistes, est la dissémination des éléments constitutifs d'une unité qui pour ainsi dire «éclate». J'en prendrai un exemple inédit dans un cahier de brouillons de I9 I 4 , le Cahier 54, pour m'interroger sur les modalités éditoriales de représentation de ce phénomène. Je précise que le repérage des événements que je vais énumérer n'aurait pas été possible sans la dimension collaborative de l'édition des Cabiers I à $75^{13}$.

Dans ces deux pages du Cahier 54 (fos $\left.\mathrm{I} 5 \mathrm{v}^{\circ}, \mathrm{I} 6 \mathrm{v}^{\circ}\right)$, Proust ébauche deux versions successives de promenades de fin de saison à Balbec en compagnie d'Albertine. Elles sont pourvues du même incipit : «L'automne vint». Dans la première, il est question des retours plus précoces à la lumière du soleil couchant qui fait «briller quelques feuillages horizontaux et clairsemés»; le second développement évoque inversement des départs plus précoces, et le simple «changement d'habitudes» qui suffit à créer un «homme différent»; il se poursuit par l'évocation d'un chêne «rose comme un pommier en fleurs», d'arbres «habillés de lierre comme des ruines». Or Proust ne va que partiellement tenir compte de la note de régie liminaire : «À propos des promenades qui figureront dans le séjour à Balbec». En effet, seule la première moitié du second développement (l'«homme différent» suscité par le «changement d'habitudes») est

I 2. Voir A. Herschberg-Pierrot, Le Style en mouvement. Littérature et art, Belin, 2005, notamment le chapitre 6 .

I 3. Je suis redevable en particulier aux transcripteurs des Cahiers 46 (J. André), 56 (P. Wise) et 73 (S.Kurokawa), ainsi qu'à $F$. Goujon, qui a repéré l'unité génétique transférée dans le Cahier 72 . 
intégrée dans le contexte prévu de la fin du second séjour à la station balnéaire, sans doute assez tardivement dans la rédaction du cahier concerné, puisqu'il s'agit, sur un recto, d'une addition à un des derniers versos (Cahier $72, \mathrm{f}^{\circ} 54 \mathrm{r}^{\circ}$ ). Le reste est disséminé, éparpillé dans d'autres promenades, à des moments distants de l'histoire d'Albertine : le chêne «rose comme un pommier en fleurs» en addition à la promenade à l'Île du Bois, entre les deux séjours à Balbec (Cahier $\left.46, \mathrm{f}^{\circ} 52 \mathrm{r}^{\circ}\right)$; les arbres «habillés de lierre comme des ruines» lors d'une promenade en automobile avec Albertine, pendant sa captivité (Cahier 73, $\mathrm{f}^{\circ}$ I $9 \mathrm{r}^{\circ}$ ), mêlés aux «allées sinueuses» et à la tonalité assombrie qu'on trouvait au début du premier morceau; enfin, le passage descriptif sur la lumière du couchant faisant «briller quelques feuillages horizontaux et clairsemés» se trouve intégré au récit d'une promenade solitaire au Bois, après la mort d'Albertine (Cahier 56 , $\left.\mathrm{f}^{\circ} 33 \mathrm{r}^{\circ}\right)$ - promenade au milieu d'«allées sinueuses», et qui rappelle au narrateur celle qu'il avait faite un jour d'hiver en compagnie de sa prisonnière.

Usage complexe, par conséquent, de deux morceaux successifs et parallèles qui se retrouvent disséminés dans pas moins de quatre cahiers différents à l'étape génétique suivante (I 9 I 5 ?), en partie d'après les injonctions partiellement contradictoires de notes de régie successives ${ }^{14}$. Le profil génétique de cette unité textuelle en deux temps ne serait pas tout à fait complet, cependant, si l'on ne précisait que le chêne «rose comme un pommier en fleurs» du Cahier 54 provient lui-même littéralement d'un ajout du Carnet $2\left(\mathrm{f}^{\circ} \mathrm{I} 8 \mathrm{r}^{\circ}\right)$, et s'est enrichi d'un «ciel bleu qui l'ensoleillait» avant de rejoindre le Cahier 46; qu'un développement rédigé dans la marge du premier morceau ( $\left.\mathrm{f}^{\circ} \mathrm{I} 5 \mathrm{~V}^{\circ}\right)$ rejoint lui aussi la promenade au Bois du Cahier 56 , sous la forme d'un ajout désormais intégré par un trait de jonction, quoiqu'encore apposé dans la marge (exemple de ce qu'on pourrait appeler une figure de montage); enfin, on remarquera qu'un élément textuel peut être disséminé plusieurs fois, puisque c'est la disposition dans deux cahiers, les Cahiers 73 et 56 , d'un même détail descriptif emprunté au Cahier 54 («les allées sinueuses» de la forêt) qui permet de faire d'une scène de promenade l'occasion, ailleurs, d'un souvenir involontaire. La place manque aussi pour aborder précisément la question du redéploiement stylistique, qui accompagne fréquemment chez Proust la reprise, rarement identique, des éléments dispersés : comparer, par exemple, «le soleil couchant faisait briller quelques feuillages horizontaux et clairsemés» (Cahier 54) avec

I 4. Première note de régie : «À propos des promenades qui figureront dans le séjour à Balbec» $\left(\mathrm{f}^{\circ} \mathrm{I} 5 \mathrm{v}^{\circ}\right)$; deuxième note de régie : «il vaudrait peut'être mieux mettre cela pour la journée dans l'île avec Albertine» ( $\left.\mathrm{f}^{\circ}{ }_{1} 6 \mathrm{v}^{\circ}\right)$; troisième note de régie : «Ces notations ne feront qu'enrichir un morceau plus consistant» (ibid.). 
«le soleil couchant faisait briller comme suspendue dans le vide, l'horizontalité clairsemée d'un feuillage d'or» (Cahier 56).

Comment représenter de tels phénomènes génétiques, qui ne sont pas rares dans les cahiers, comme n'est pas rare la figure inverse, celle du montage, où des fragments empruntés à divers folios d'un même cahier (ou de plusieurs cahiers) se combinent sur une même page ou double page? L'annotation de l'édition imprimée se borne ici nécessairement au recensement des événements d'écriture, et limite le commentaire à l'essentiel. De toute évidence, les diagrammes génétiques, tracés avec précision sur les transcriptions diplomatiques correspondantes, peuvent se révéler plus «parlants» (fig. 2 et 3, p. I 66).

On pourrait envisager d'«animer» ces cartes par une série de liens hypertextuels, pour circuler de transcription en transcription. Les diagrammes génétiques pourraient d'ailleurs être réalisés non seulement à partir des transcriptions mais aussi à partir des fac-similés, avec l'enrichissement d'un zonage coloré, permettant de repérer instantanément les différentes unités génétiques (fig. 4, p. I 67). Grâce à un système de calques, on devrait pouvoir ainsi, en déplaçant la souris sur la transcription diplomatique ou sur le facsimilé «nus», faire apparaittre, au choix, le diagramme des unités textuelles ou celui des unités génétiques... puis, pour ce dernier, les unités qui en dépendent.

Toutefois, la petite maquette présentée ici a négligé un aspect crucial de l'analyse génétique, à savoir la chronologie de la dissémination, et donc n'a pas pris en charge la question de sa représentation : en mettant tous les événements d'écriture sur le même plan tabulaire, on a opéré, il faut bien le reconnaitre, une description plus qu'un classement.

Il a bel et bien existé un ordre, et un seul, dans lequel se sont progressivement remplies les milliers de pages des cahiers d'À la recherche du temps perdu. Mais au vu du nombre très élevé des événements de genèse textuelle que l'édition des Cahiers I à 75 , et ses prolongements mettent et vont continuer à mettre en évidence, la question de la possibilité même d'un classement chronologique relatif de tous les événements d'écriture se pose (on entrevoit d'ailleurs, au vu de l'exemple proposé, la difficulté de l'exercice sur une fraction même minime du corpus ${ }^{15}$ ).

I 5 . Le travail de classement le plus fouillé, qui reprend et discute ceux des prédécesseurs, est la somme d'A. Pugh, The Growth of À la recherche du temps perdu. A Chronological Examination of Proust's Manuscripts from Igog to I9I4, Toronto, University of Toronto Press, 2004, deux volumes. Toutefois, en ce qui concerne le Cahier 54 (I9I4), seule la première unité textuelle est évoquée. 


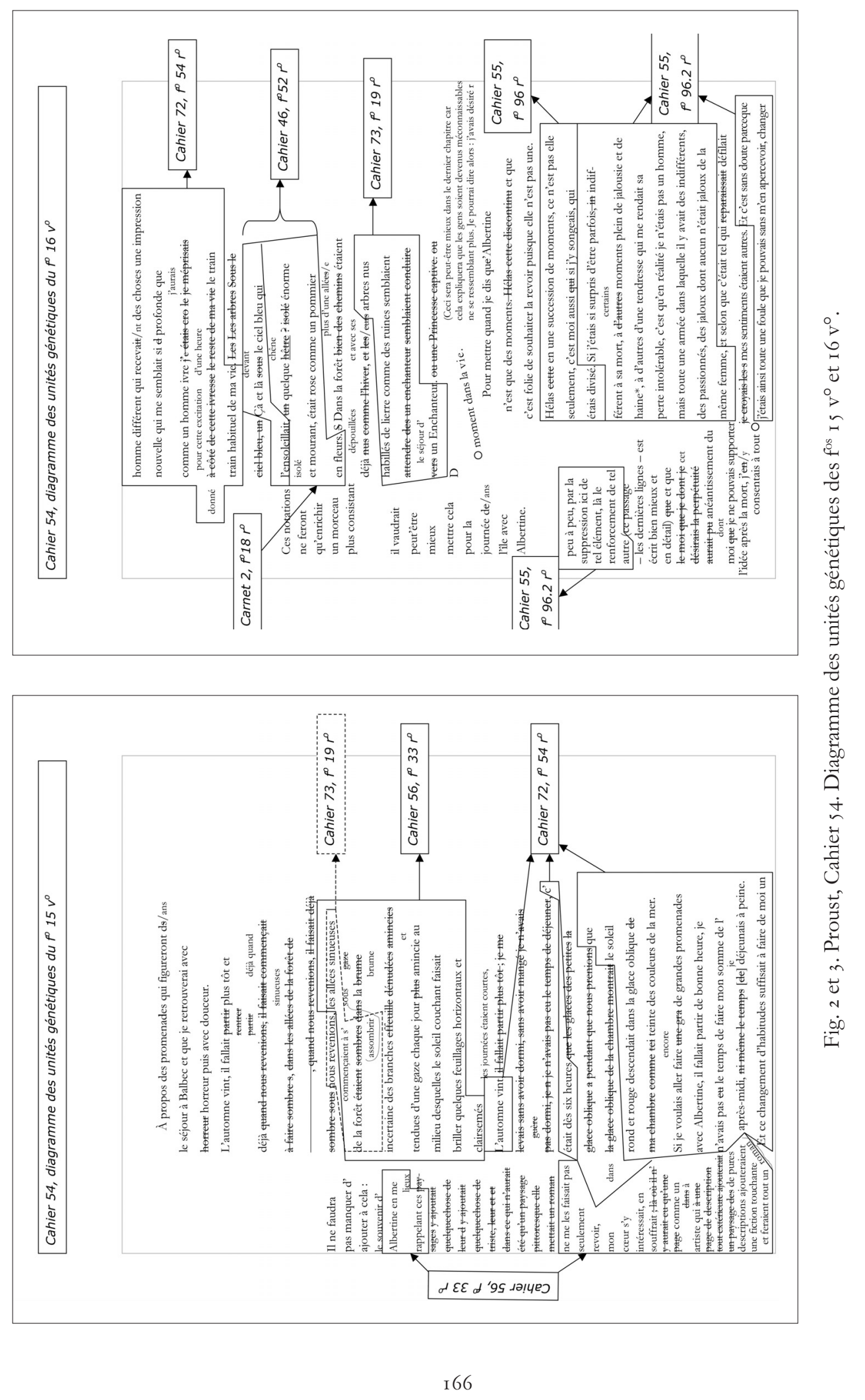



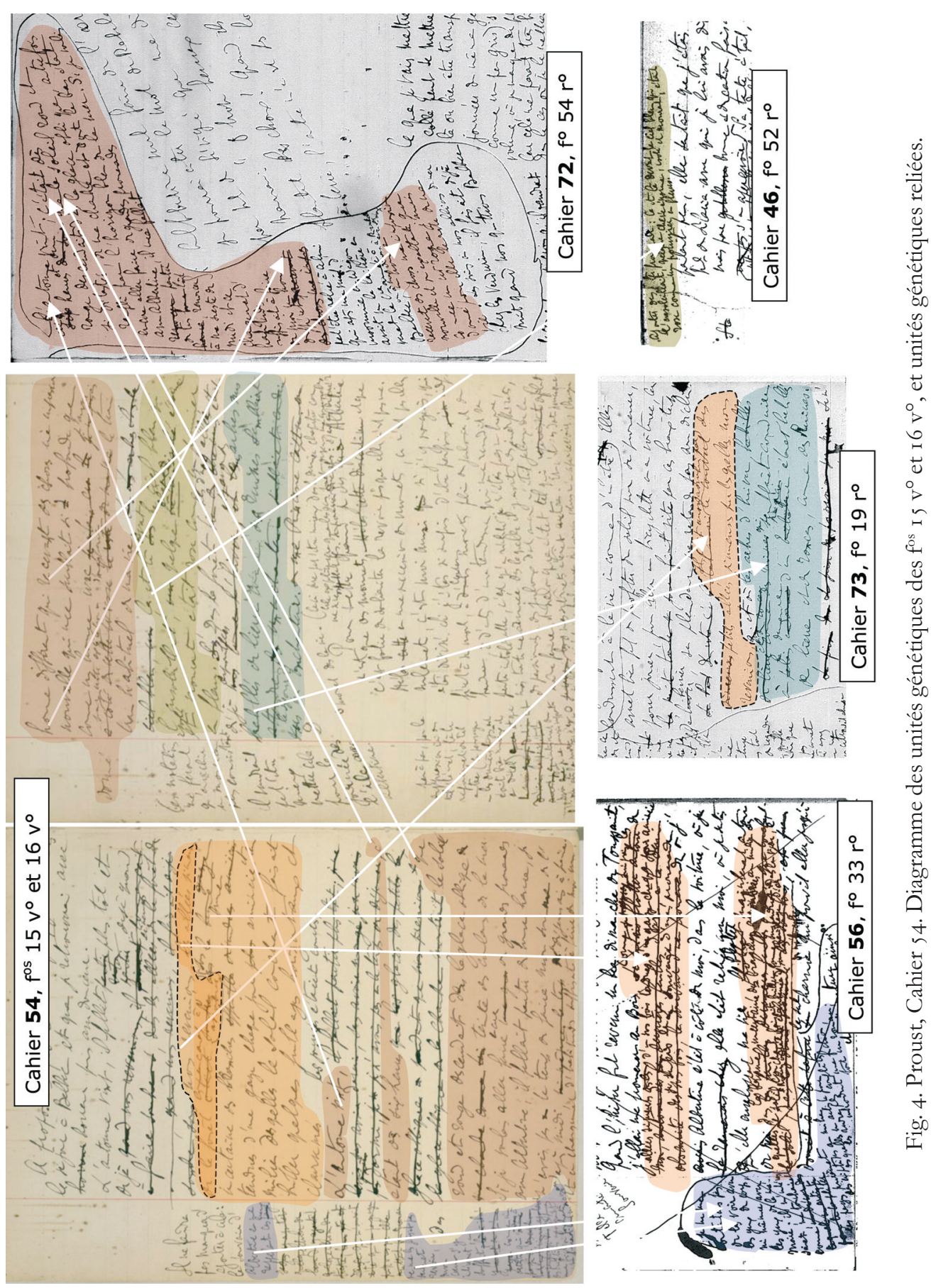
Faut-il envisager des éditions hypertextuelles fragmentaires? C'est déjà le cas dans le cadre du programme de recherche OPTIMA ${ }^{16}$, qui réunit un laboratoire littéraire, l'ITEM-CNRS, deux laboratoires informatiques (LIPN, Paris-Nord; LITIS, Rouen) et la Bibliothèque nationale de France autour d'un projet de plate-forme éditoriale en ligne de manuscrits de Flaubert, Proust et Valéry. Pour l'axe proustien, le projet concerne les cahiers dits «d'Albertine» (I9I 3-I9I 5), et quelques-uns de leurs antécédents parmi les cahiers des Jeunes filles (I 909-I 9 I I). En deçà même de la question du classement des unités génétiques et de la définition de l'interface pour l'hypertexte, un des enjeux les plus délicats du projet est la mise au point d'un logiciel de transcription qui parvienne à concilier l'encodage du document (description physique, événements d'écriture : ajouts, biffures, etc.) avec un affichage diplomatique satisfaisant. C'est encore un écueil pour l'édition électronique, comme, en miroir, une des limites les plus manifestes de l'édition imprimée est l'impossibilité d'une recherche lexicale ou thématique automatisée dans le corpus des transcriptions (insuffisance qu'un hyper-index nominum ne pallierait que très partiellement).

\section{Conclusion provisoire}

Bien entendu, un «authentique» Hyper-Proust inclurait non seulement les soixante-quinze cahiers, mais l'ensemble du fonds Proust de la Bibliothèque nationale de France; et non seulement l'ensemble du fonds Proust, mais le texte d'À la recherche du temps perdu publié par Proust, jusqu'à Sodome et Gomorrhe II (1 922), et problématiserait la question de l'édition de la fin du roman, laissée à l'état de chantier manuscrit; un Hyper-Proust enfin devrait mettre à disposition non seulement l'ensemble des manuscrits et le texte définitif correspondant quand il existe, mais le considérable corpus épistolaire, sans compter la «bibliothèque» proustienne, entendue au sens le plus large, dans toutes ses rubriques, notamment musicale et picturale. Pour le moment, je me suis contentée d'entendre par Hyper-Proust, on l'aura compris, la mise en relation systématique et raisonnée de toutes les transformations textuelles dans le corpus des Cahiers I à 75 , élargi quand c'est nécessaire au reste du corpus manuscrit, ainsi qu'au texte définitif.

L'annotation génétique, les index, l'Hyper-index et les diagrammes génétiques constituent donc l'Hypo-Proust de cet Hyper-Proust génétique. S’ils

I6. Outils Pour le Traitement et l'analyse de l'Information dans les MAnuscrits modernes (financement ANR; pilotage scientifique de P.-M. de Biasi, ITEM). Le projet comporte également un axe Braudel (MSH, Paris). 
fournissent le matériau de l'hypertexte, ils n'en précisent pas toutefois la physionomie future. S'agit-il de produire une «simple» édition destinée à la consultation, ou un opérateur de savoir, autrement dit un instrument de «fouille» qui permettra à l'utilisateur de contribuer au recensement de toutes les unités génétiques, et, peut-être, d'aider à leur classement? D’un point de vue tout pratique, comment résoudre le conflit entre une mémoire virtuellement infinie, celle du disque dur, avec l'exiguiité du «bureau» électronique, qui fait que seule une infime partie de cette mémoire infinie virtuellement disponible peut en réalité, à un moment n, être mobilisée, présentifiée? Ainsi, loin d'être seulement un instrument pour l'édition électronique (un pis-aller, en «attendant mieux»!), l'édition imprimée en demeurera-t-elle longtemps l'indispensable complément (et vice-versa). Il serait sans doute fructueux, d'ailleurs, d'envisager l'élaboration de l'hypertexte électronique dans la complémentarité directe de l'édition imprimée.

Revenons pour finir aux enjeux intellectuels et peut-être théoriques d'un Hyper-Proust. L'existence d'un hypertexte électronique reviendrait pratiquement à rendre caduque une forme de discours critique encore prévalente, celle du «récit de genèse», en particulier dans sa variante à prétention «totalisante»: le remplaceront des séries de parcours génétiques (plus ou moins exhaustifs en fonction des documents disponibles), parcours qui se croiseront, se chevaucheront en partie, ouvrant de proche en proche à tout l'éventail de la genèse. D'autre part, dans la mesure où l'essence de l'hypertextualité est le saut, le détour, la non-linéarité, on ne saurait exiger de l'utilisateur de l'Hyper-Proust qu'il se contente des parcours génétiques tout faits qu'on lui proposera ou qu'il établira. L'hypertexte virtuel de l'édition imprimée dessine déjà en pointillé des réseaux qui ne cessent de se croiser avec d'autres réseaux, montrant qu'en tout point ou presque, in fine, une bifurcation sera possible - et même : tentante-d'un «nœud» d'un parcours génétique (une zone de folio) vers le nœud d'un autre parcours (une zone voisine du même folio...). Même s'il s'agit, avec la mise au point d'un hypertexte électronique, de donner une représentation vectorisée des transformations textuelles successives, rien n'empêchera d'en faire un usage lui-même bypertextuel, c'est-à-dire non-linéaire, irrespectueux de la logique même des parcours. Certes, il y a là le risque de désorientation pure et simple caractéristique de l'usage «sauvage» des réseaux, mais il se pourrait aussi que cet usage transversal soit lui-même heuristique : il y aura sans doute une sérendipidité une possibilité de découverte imprévue et féconde... - dans ces explorations futures.

Avec un possible Hyper-Proust, il ne s'agira donc plus seulement pour l'utilisateur d'être dehors (position critique traditionnelle) mais dedans, immergé, 
pour mener, sans vectorisation, des trajets inédits - trajets zigzagants peutêtre, fictionnels probablement, au sens où ils ne correspondront plus à aucun parcours dûment identifiable du point de vue génétique ${ }^{17}$. Scandale? Ou simple effet boomerang : le schéma réticulaire de l'écriture proustienne (si bien pressenti par Gilles Deleuze : «Une œuvre qui n'est pas une robe, qui n'est pas une cathédrale, mais une toile d'araignée en train de se tisser sous nos yeux $\left.{ }^{18} »\right)$ pourrait bien finir par nous envelopper.

17. Voir N. Mauriac Dyer, «Déchiffrer. Transcrire. Cartographier. Lier. Proust ou le manuscrit apprivoisé», Genesis, n 27, 2006, p. 2 I-36.

I 8. Cabiers Marcel Proust 7, Etudes proustiennes II, Gallimard, i975, p. 9I : extrait d'une «Table ronde» avec R. Barthes, G. Genette et al., dirigée par S. Doubrovsky, lors du colloque «Proust et la nouvelle critique» du 20 au 22 janvier 1972 (Paris, ENS); texte revu et mis au point par J. Bersani. 